\title{
Persistência do ducto arterioso em cães: revisão
}

\section{Patent ductus arteriosus in dogs: a review}

\section{Persistencia del conducto arterioso en perros: revisión}

\author{
Angelo João Stopiglia; ${ }^{1}$ Rodrigo Ramos de Freitas; ${ }^{2}$ Eduardo Toshio Irino; ${ }^{3}$ Maria Helena \\ Matiko Akao Larsson; ${ }^{4}$ Fabio Biscegli Jatene ${ }^{5}$
}

Laboratório de Cirurgia Cardiotorácica do Departamento de Cirurgia da Faculdade de Medicina Veterinária e Zootecnia da Universidade de São Paulo (FMVZ/USP). São Paulo, SP, Brasil

\section{Resumo}

Objetivo: Apresentar revisão da literatura sobre a persistência do ducto arterioso, levantando os principais aspectos morfológicos, fisiopatológicos, clínicos, de diagnóstico e prognóstico, bem como os possíveis tratamentos. Fontes Consultadas: CAB Abstracts 1990-2002; MEDLINE 1978-2002. Síntese dos Dados: A persistência do ducto arterioso (PDA) é a doença cardíaca congênita encontrada com maior freqüência em cães. A forma mais comum é caracterizada pelo desvio sanguíneo da esquerda para a direita, embora, mais raramente, presencie-se a PDA reversa, ou seja, com inversão do fluxo através do ducto. Conclusões: $\mathrm{O}$ entendimento conjunto de todos estes aspectos auxilia na compreensão dos fenômenos envolvidos na evolução clínica da afecção, contribuindo de maneira decisiva para a indicação do tratamento, visando à recuperação do animal.

Palavras-chave: Cardiopatias congênitas. Persistência do conduto arterioso. Cirurgia torácica. Cães.

'Professor Titular do Departamento de Cirurgia e Coordenador do Laboratório de Cirurgia Cardiotorácica do Departamento de Cirurgia da FMVZ/USP. CRMV-SP 1589

${ }^{2}$ Pós-Graduação, nível Doutorado, do LCCT/VCI da FMVZ/USP. CRMV-SP 8522

${ }^{3}$ Pós-Graduação, nível Mestrado, do LCCT/VCI da FMVZ/USP. CRMV-SP 15821

${ }^{4}$ Professora Titular do Departamento de Clínica Médica da FMVZ/USP. CRMV-SP 0796

${ }^{5}$ Professor Associado do Departamento de Cardiopneumologia da Faculdade de Medicina da USP. CRM-SP 33865 


\section{Introdução}

A persistência do ducto arterioso (PDA), também denominada de ducto arterioso patente é o defeito cardíaco congênito mais freqüentemente encontrado em cães. Com relação aos gatos, pode-se afirmar que sua ocorrência seja rara (COTE; ETTINGER, ${ }^{1}$ 2001; FOSSUM, ${ }^{2}$ 1997; JONES; BUCHANAN, ${ }^{3}$ 1981; OSWALD; ORTON, ${ }^{4} 1993$; SNAPS et al., ${ }^{5}$ 1995; TIDHOLM,${ }^{6}$ 1997).

Existem duas condições com características hemodinâmicas diversas em relação à persistência do ducto arterioso: a primeira, a mais freqüente, é caracterizada pelo fluxo sanguíneo no interior do ducto, ocorrendo no sentido da esquerda para direita, ou seja, da aorta para o tronco pulmonar, denominada de persistência do ducto arterioso clássica (PDAc); a segunda forma, chamada persistência do ducto arterioso reverso (PDAr), caracteriza-se pela reversão do fluxo sanguíneo, isto é, no sentido do tronco pulmonar para a aorta.

Autores relatam que tal cardiopatia acomete principalmente as raças Maltês, Lulu da Pomerânia, Chihuahua, Pastor de Shetland, English Springer Spaniels, Keeshound, Bichon Frisé, Poodle e Yorkshire Terrier, observando-se mais predisposição em fêmeas na proporção de 1,72:1 (FOSSUM, ${ }^{2}$ 1997; ACKERMAN et al., ${ }^{7}$ 1978; BIRCHARD; BONAGURA; FINGLAND,${ }^{8}$ 1990; BONAGURA, ${ }^{9}$ 1992; EYSTER, ${ }^{10}$ 1993;).

A principal conseqüência da PDAc no coração é a sobrecarga do ventrículo esquerdo, o que incorrerá em processo de dilatação e hipertrofia ventricular esquerda. Secundariamente a esse processo, está a distensão do anel da valva atrioventricular esquerda, causando regurgitação. Fibrilação atrial pode ocorrer ainda como seqüela da dilatação atrial esquerda. ${ }^{2,3}$

Devido ao aumento do fluxo sanguíneo no pulmão em cães com PDAc, com freqüência, é observado edema pulmonar. Raramente, podem desenvolver hipertensão pulmonar suprasistêmica que reverte a direção do fluxo no ducto, causando a chamada
PDAr e culminando em grave hipoxemia e cianose. Normalmente, tal quadro é decorrente da nãocorreção cirúrgica do defeito. . $^{1,2,10}$

De modo geral, a PDA não vem acompanhada de outros defeitos congênitos, embora alguns autores relatem casos de PDA associados à persistência de arco aórtico direito com posterior obstrução esofágica (HOLT et al., ${ }^{11}$ (2000), tetralogia de Fallot (McENTEE et al., ${ }^{12}$ 1998), persistência de veia cava cranial e displasia de valva atrioventricular direita (FERNANDEZ DEL PALACIO et al., ${ }^{13}$ 1997).

\section{Morfologia}

O ducto arterioso é um vaso derivado do sexto arco aórtico esquerdo existente durante a vida fetal, que conecta o tronco pulmonar à aorta, obliterandose após o nascimento pela reorganização histológica da parede do ducto, quando então passa a ser chamado de ligamento arterioso, segundo Bonagura ${ }^{9}$ (1992), Ettinger e Sutter ${ }^{14}$ (1970) e Pyle et al. ${ }^{15}$ (1981).

O mecanismo pelo qual realiza-se a oclusão do ducto arterioso ainda não está bem esclarecido. Acredita-se que logo após o parto a tensão do oxigênio leva à inibição das prostaglandinas locais, promovendo a oclusão funcional do ducto e, mais tardiamente, progredindo para a obliteração anatômica nas próximas semanas de vida. ${ }^{9}$ Dentre as transformações histológicas que ocorrem durante a oclusão fisiológica do ducto observa-se, primeiramente, a separação de células endoteliais da lâmina elástica interna, resultando em amplo edema subendotelial; a seguir, constata-se a migração de fibras musculares lisas não-diferenciadas e aposição de células endoteliais no interior do lúmen do ducto. Assim, para De Reeder et al. ${ }^{16}$ (1989) e Gittenberger De Groot et al..$^{17}$ (1985), tais transformações não são verificadas no quadro de PDA. A persistência do ducto arterioso é explicada como resultado de modificações histológicas no interior da parede do ducto, notadamente das fibras musculares. Enquanto a parede normal do ducto arterioso possui padrão 
frouxo de musculatura lisa circunferencial em cães normais, a parede do ducto arterioso persistente apresenta estrutura não-contrátil remanescente da aorta, deixando sua parede mais rígida, prejudicando sensivelmente a sua capacidade de sofrer a oclusão fisiológica. ${ }^{9} \mathrm{O}$ resultado de tal processo é a persistência de ducto arterioso (Figura 1).

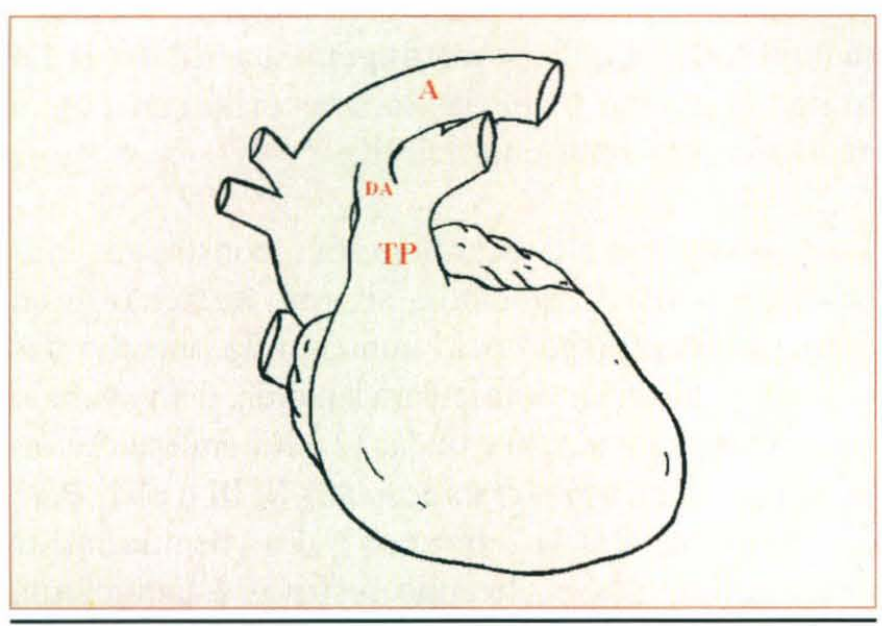

Figura 1 - Esquema com a localização anatômica do ducto arterioso. $\mathrm{A}=$ aorta, $\mathrm{TP}=$ tronco pulmonar, $\mathrm{DA}=$ ducto arterioso

O que se tem observado usualmente em cães, é que o ducto arterioso persistente é largo, sendo maior que $1 \mathrm{~cm}$ de diâmetro, porém curto, com aproximadamente $1 \mathrm{~cm}$ de comprimento, localizado entre a aorta e o tronco pulmonar, caudal à origem do tronco braquicefálico e artéria subclávia esquerda., 2,10

\section{Fisiopatologia}

Durante a vida fetal, a pressão na artéria pulmonar é maior que a pressão sistêmica, o que direciona naturalmente o fluxo sanguíneo no interior do ducto arterioso da direita para a esquerda. Após o nascimento, a resistência vascular sistêmica repentinamente aumenta devido à eliminação da baixa resistência da circulação placentária. Concomitantemente, a resistência vascular pulmonar diminui devido à expansão dos alvéolos e do efeito do aumento da $\mathrm{pO}_{2}$, causando dilatação vascular pulmonar. Nessas condições, caso o ducto arterioso persista, observa-se a forma clássica de PDA, com desvio do fluxo sanguíneo da aorta para o tronco pulmonar. ${ }^{15}$
Tal desvio vai resultar em contínuo sopro cardíaco, aumento do fluxo pulmonar e aumento do retorno venoso para o átrio e o ventrículo esquerdos. Tanto na aorta quanto no tronco pulmonar, dependendo da forma de persistência de ducto, pode-se encontrar dilatação destas estruturas. ${ }^{9}$

A persistência do ducto arterioso pode resultar em duas condições clínicas distintas: primeiramente, se aquele permanecer patente com resistência vascular pulmonar normal ou próximo ao normal, a pressão suprasistêmica vai se sobrepor à pressão no tronco pulmonar e será criado o PDAc, com desvio da aorta para o tronco pulmonar., ${ }^{45}$ Caso a resistência vascular pulmonar fetal seja mantida, a pressão no tronco pulmonar será próxima ou excederá a pressão na aorta. O resultado desse processo será traduzido por desvio bidirecional ou desvio do tronco pulmonar para a aorta, denominada de PDAr. ${ }^{4,10,15}$

Decorrente do fato de que o ducto arterioso persistente localiza-se caudalmente ao tronco braquiocefálico e à artéria subclávia esquerda e, portanto, que o desvio sanguíneo será efetuado após aquele já ter sido direcionado para os referidos vasos, observa-se, em alguns casos, o quadro de cianose na metade caudal do corpo do animal visualizado principalmente na mucosa vaginal ou peniana. ${ }^{15}$

No caso de PDAr, observa-se policitemia secundária em resposta ao aumento da eritropoetina devido à hipoxemia crônica. Uma das conseqüências hemodinâmicas desta policitemia é o aumento da viscosidade sanguínea, levando ao quadro de insuficiência cardíaca congestiva ou trombose intravascular, com infarto de vários órgãos. ${ }^{10,15}$

\section{Sinais Clínicos}

Na PDAc, os animais podem não apresentar sinais clínicos durante um certo período de tempo, segundo van Israel et al. ${ }^{18}$ (2001), como pode também apresentar intolerância a exercício, como tosse; para Fossum ${ }^{2}$ (1997), Snaps et al..$^{5}$ (1995), Eyster ${ }^{10}$ (1993), O'Brien et al..$^{19}$ (1998) e Saunders et al. ${ }^{20}$ 
(1999), fraqueza em membros pélvicos e retardo no crescimento. $^{15}$

Ao exame físico, durante auscultação do tórax, nos casos de PDAc, percebe-se sopro cardíaco de "maquinaria" contínuo, acentuado durante sístole e audível principalmente na região de base esquerda do coração, como relatam Fossum ${ }^{2}$ (1997), Snaps et al. ${ }^{5}$ (1995), Saunders et al. ${ }^{20}$ (1999) e Henderson e Jackson ${ }^{21}$ (1983), sendo de grande intensidade, normalmente se sobrepondo aos sons normais do coração. ${ }^{10,15} \mathrm{O}$ pulso femoral é forte e hipercinético. As membranas mucosas ainda permanecem normocoradas, tornandose cianóticas apenas quando se instaura quadro de insuficiência ventricular e edema pulmonar.?

Já na PDAr nota-se dispnéia, apatia, síncope (ARORA, ${ }^{2}$ 2001; BONAGURA, ${ }^{9}$ 2002) e à semelhança da PDAc, também é visto intolerância ao exercício e fraqueza na sustentação de membros pélvicos após exercício. ${ }^{1,4,15}$ Durante auscultação do tórax, não são criadas turbulências significativas na luz do ducto arterioso capazes de produzir sopros fortes. ${ }^{15}$ Nestes casos especificamente nota-se desdobramento da segunda bulha cardíaca. Em raros casos constata-se sopro diastólico de insuficiência pulmonar devido à hipertensão pulmonar. ${ }^{21}$

Os achados de exame físico em animais com PDAr são principalmente cianose diferencial, evidente na parte caudal do corpo do animal, devido à mistura de sangue da aorta, oxigenado, com o sangue do tronco pulmonar ainda não oxigenado. Essa mistura, como já explicado, ocorre caudalmente ao tronco braquiocefálico e artéria subclávia esquerda, o que justifica a mucosa oral e ocular apresentarem-se normocoradas. ${ }^{2,4}$

Hipoxemia sistêmica estimula a produção de eritrócitos levando à policitemia secundária, exteriorizada pelo quadro sintomático de fadiga, dispnéia, taquicardia e fraqueza de membros pélvicos. Episódios de síncope e incoordenação podem ocorrer em decorrência da hiperviscosidade do sangue, que na microvascularização acarreta hipóxia de cérebro e de outros tecidos. ${ }^{1,4,22}$

\section{Diagnóstico}

Os principais achados nas radiografias torácicas em casos de PDAc são hipervascularização pulmonar, aumento ventricular e atrial esquerdo, dilatação dos vasos pulmonares e edema alveolar e intersticial, ${ }^{2,7,9,20}$ como também pode ser notado aumento ventricular direito. ${ }^{10,15}$ Já os achados radiográficos da PDAr restringem-se à dilatação do tronco pulmonar e artérias lobares principais, além de hipertrofia ventricular direita. .,9,19,22 $^{2}$

Ao exame eletrocardiográfico constata-se, nos casos de PDAc, taquicardia sinusal, aumento atrial esquerdo identificado pelo aumento da duração das ondas P e dilatação ventricular esquerda, demonstrado por eixo frontal normal e ondas Q e R com maior voltagem nas derivações craniocaudais II, III e aVF. Para o caso de PDAr, poucos são os achados eletrocardiográficos, ficando restritos à taquicardia sinusal, desvio de eixo que sugere déficit no sistema de condução e hipertrofia ventricular direita. , $^{5,10,20-22}$

O exame ecocardiográfico fornece o diagnóstico definitivo para ambos os casos de PDA. Para o PDAc pode-se visualizar aumento atrial esquerdo, dilatação e hipertrofia ventricular esquerda, fluxo turbulento de ejeção da aorta, além de poder elucidar o diâmetro e comprimento do ducto, constituindose informações importantes para o cirurgião. ${ }^{13,21} \mathrm{Com}$ exame dopplercardiográfico é possível identificar a direção do fluxo no ducto arterioso e a presença de insuficiência pulmonar em casos de PDAr. Nestes casos de PDAr notam-se hipertrofia ventricular direita e dilatação do tronco da pulmonar., ${ }^{9,21,22} \mathrm{O}$ exame ecocardiográfico também pode ser útil para descartar outros defeitos cardíacos concomitantes, de acordo com Moise ${ }^{23}$ (1989).

Alterações laboratoriais normalmente não são encontradas no quadro de PDAc. No entanto, quando o caso se refere à PDAr, comumente encontra-se policitemia em resposta ao aumento da eritropoetina devido à hipoxemia crônica. É de importância ímpar saber proceder ao diagnóstico diferencial com outras afecções que denotam semelhanças à auscul- 
tação, como casos de estenose de aorta ou defeito do septo interventricular, cujos sopros são parecidos aos do quadro de PDAc. Já a PDAr pode ser confundida, em muitos achados, com a tetralogia de Fallot, defeito do septo interventricular e defeito do septo interatrial. $\mathrm{O}$ aumento de ventrículo esquerdo e do tronco pulmonar também não deve ser confundido com estenose de valva pulmonar ou quadro de vermes pulmonares (dirofilária)., ${ }^{2,15}$

\section{Tratamento}

Na PDAc, o tratamento de eleição é a correção cirúrgica, porém, deve-se atentar primariamente para o estado geral do paciente. Para aqueles que apresentem quadro de edema pulmonar, deve-se instituir tratamento à base de furosemida antes da intervenção cirúrgica. Nos casos em que for detectada fibrilação atrial, a atividade cardíaca deve ser monitorada e, se necessário, administrar inotrópicos positivos, como digoxina (FOSSUM, ${ }^{2}$ 1997; EYSTER, ${ }^{10} 1993$; SAUNDERS et al., ${ }^{20}$ 1999; DOWNS; STAMPLEY; RAWLINGS, ${ }^{24}$ 1995; HUBER; MONTAVON, ${ }^{25}$ 1992).

Animais com sinais de insuficiência cardíaca congestiva devem ser tratados com inotrópicos positivos (digoxina), vasodilatadores (maleato de enalapril) e diuréticos (furosemida). Excesso de diuréticos e/ou vasodilatadores podem levar à hipotensão, devendo, portanto, ser administrados com cautela. ${ }^{2}$

Adequada avaliação pré-operatória é fator crucial para o sucesso da intervenção cirúrgica. Para Fantoni $^{26}$ (2002), todos os dados do histórico clínico do animal, exame físico e os laudos dos exames complementares, como o eletrocardiograma, radiografia torácica, ecocardiograma e análise laboratorial devem estar em poder do anestesista, a fim de que possa avaliar o quadro e proceder à conduta mais apropriada para o caso.

Estabilizado o quadro do paciente no período pré-operatório, procede-se à intervenção cirúrgica, que terá como objetivo a ligadura do ducto arterioso persistente. Como medicação pré-anestésica, o recomendado é a administração de morfina ou meperidina. Para a indução, o propofol e a manutenção com isofluorano. A conduta anestésica deve ter monitorização invasiva de pressão arterial, visto que duas das principais intercorrências transoperatórias são hipertensão e hemorragia. ${ }^{26}$

O animal é posicionado em decúbito lateral direito e submetido à preparação de campo cirúrgico. Realiza-se toracotomia intercostal esquerda, no quarto espaço intercostal. Posiciona-se o afastador de Finochietto prosseguindo com o rebatimento caudal dos lobos pulmonares com gaze umedecida para expor a área referente ao ducto arterioso, na qual será incisado o saco pericárdico paralelamente e dorsal ao nervo frênico ${ }^{2,10,24}$ (Figuras 2 e 3).

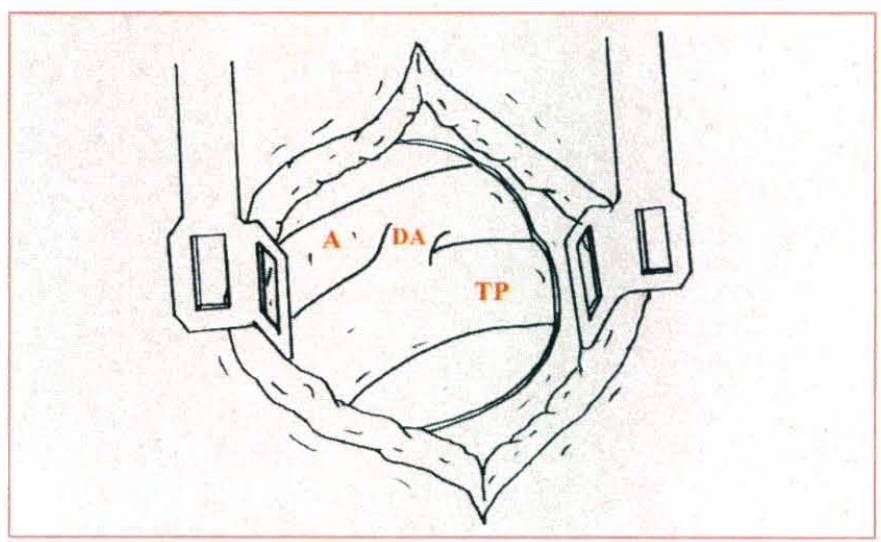

Figura 2 - Esquema em que visibiliza-se o campo cirúrgico após a dissecção do ducto arterioso. $\mathrm{A}=$ aorta, $\mathrm{TP}=$ tronco pulmonar, $\mathrm{DA}=$ ducto arterioso

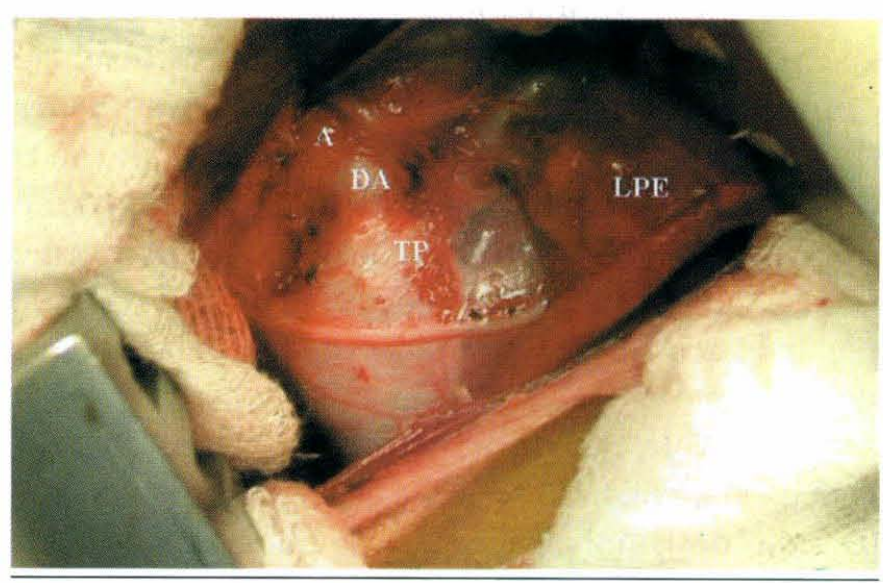

Figura 3 - Cão da raça Poodle, 1 ano, apresentando persistência de ducto arterioso. $\mathrm{A}=$ aorta, $\mathrm{DA}=$ ducto arterioso, $\mathrm{TP}=$ tronco pulmonar, LPE = lobo pulmonar esquerdo 
Conforme Fossum, ${ }^{2}$ EYSTER ${ }^{10}$ e GOODWIN, ${ }^{27}$ o nervo vago posiciona-se dorsalmente ao ducto arterioso persistente, devendo ser identificado e reparado durante a dissecção do ducto. A técnica mais utilizada na correção da PDAc é a dissecção do ducto arterioso e sua posterior dupla ligadura.

Para proceder a dissecção ao redor do ducto arterioso utilizam-se pinça Mixter ou pinça de Meeker reta (Figura 4). Nos casos de animais de pequeno porte, pode-se utilizar uma pinça Halsted para realizar a dissecção bem como para a passagem do fio. ${ }^{21}$

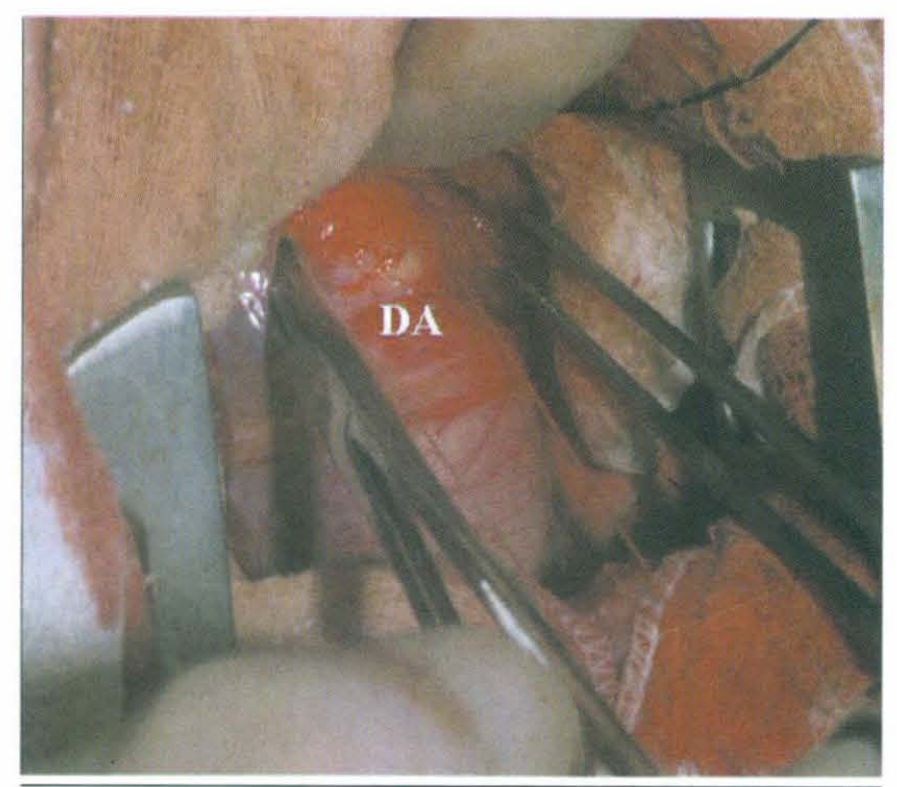

Figura 4 - Figura ilustrando fio de sutura sendo carreado ao redor do ducto com auxílio de pinça Mixter. DA = ducto arterioso

Com a própria pinça Mixter ou com a utilização de um carreador de ligadura medialmente ao ducto arterioso é passado fio de sutura (polipropileno 4-0 ou seda 1-0) de modo que, após a retirada da pinça, o fïo posicione-se junto ao ducto, em sua face medial (Figura 5). Normalmente são realizadas duas laçadas para reduzir o risco de recanalização, sendo primeiramente ligada à região do ducto próximo à aorta (Figura 6). Neste momento, a pressão arterial aumenta significantemente, concomitante com a redução na freqüência cardíaca (reflexo de Branham). O tronco pulmonar diminui seu tamanho, e após um a dois minutos é aplicado o nó à segunda ligadura, próximo ao tronco pulmonar. $^{2,10,21,24}$ (Figura 6).

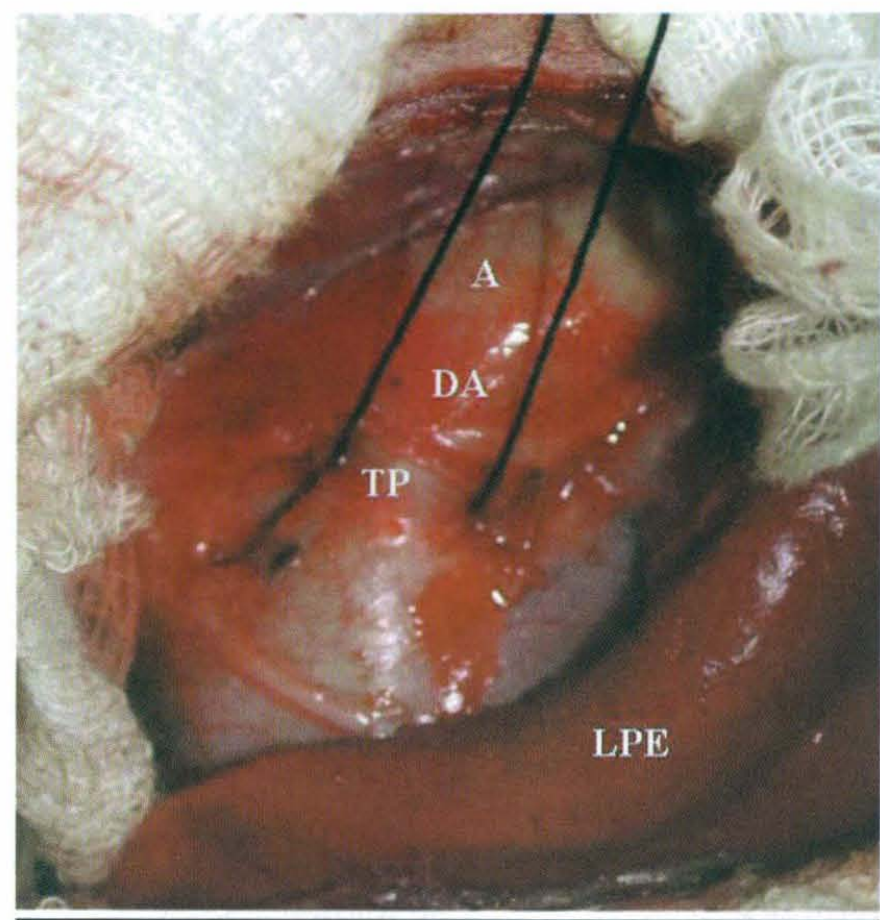

Figura 5 - Cão da raça Poodle, 1 ano, apresentando persistência de ducto arterioso. $\mathrm{A}=$ aorta, $\mathrm{TP}=$ tronco pulmonar, $\mathrm{LPE}=$ lobo pulmonar esquerdo. Fio de sutura ao redor do ducto arterioso (DA)

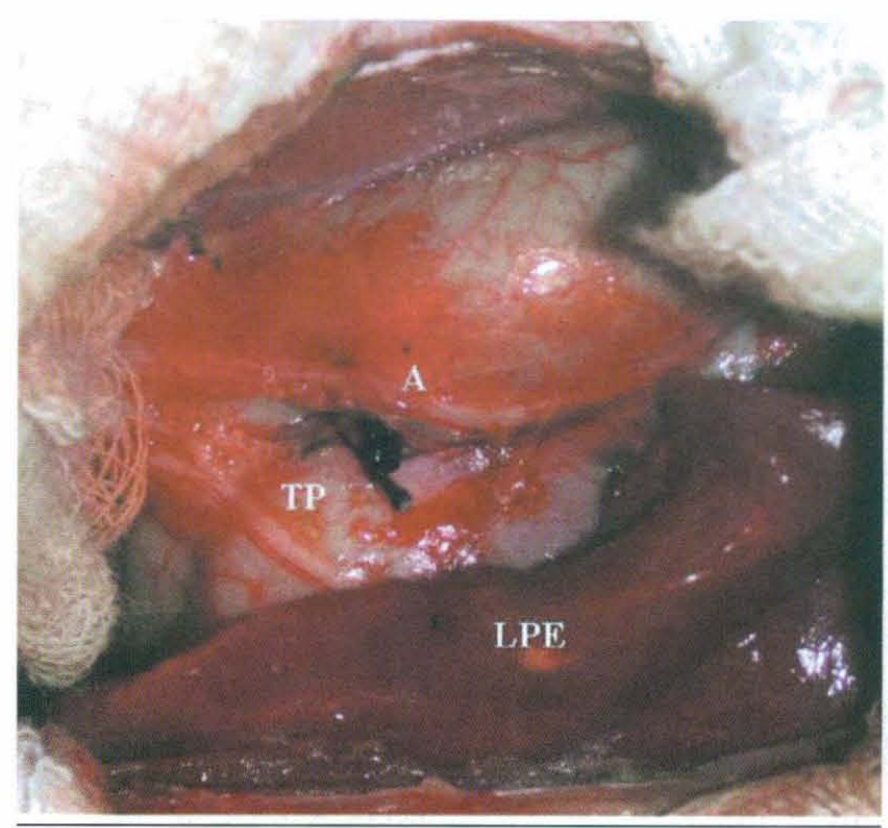

Figura 6 - Cão da raça Poodle, 1 ano, apresentando persistência de ducto arterioso. Ducto arterioso após dupla ligadura. $\mathrm{A}=$ aorta, $\mathrm{TP}=$ tronco pulmonar, $\mathrm{LPE}=$ lobo pulmonar esquerdo

Finalizada a ligadura do ducto, coloca-se dreno torácico temporário do tipo selo d'água de modo a restabelecer pressão negativa intratorácica. Procede-se a seguir a toracorrafia, com aproximação dos arcos 
costais e sutura de músculos de parede torácica e pele. Com a estabilização mecânica da ferida cirúrgica, aplicam-se curativos estéreis compressivos na região..$^{21,24}$

Cuidados pós-operatórios devem ser tomados, principalmente para controlar a dor por meio de analgésicos, além de curativo na ferida cirúrgica, atentando para que aquele não dificulte o trabalho respiratório do animal. ${ }^{21}$ Cloridrato de bupivacaína intercostal ou intrapleural são usados para analgesia suplementar. $^{2}$

A sutura realizada na pele do animal é retirada entre sete e dez dias após o ato operatório. ${ }^{10}$

Nos casos de PDAr, a correção cirúrgica por meio de ligadura e fechamento do ducto é contraindicada. Tal circunstância decorre do fato de que o ducto atua como uma valva de alívio, prevenindo o excesso de pressão sistêmica sobre o coração direito. Caso o ducto seja ligado, acentuada hipertensão pulmonar vai culminar em sobrecarga da porção direita e conseqüente insuficiência cardíaca. ${ }^{22}$

Portanto, o tratamento consiste em cuidados de manejo com o animal, mantendo-o em repouso, evitando situações de estresse e restringindo exercícios. Deve-se também manter o hematócrito entre 62 e $68 \%$. Pode-se proceder a flebotomia, porém, sempre mediante infusão contínua de soluções cristalóides. ${ }^{1,9,21}$.

Tem-se relatado outras alternativas que visam ao tratamento de policitemia secundária nos casos de PDAr, com o uso de drogas supressoras da eritropoiese como a hidroxiurea e a ciclofosfamida. No entanto, deve-se atentar para os efeitos tóxicos que tais drogas acarretam, principalmente na medula óssea (COTE; ETTINGER, ${ }^{1}$ 2001; MOORE, ${ }^{28}$ 2001).

Outras técnicas de fechamento de PDAc têm sido descritas na literatura. Algumas delas utilizam cateterização, antes somente aplicada em medicina humana. Tal procedimento cirúrgico, segundo Snaps et al. ${ }^{5}$ (1995) e Schneider et al. ${ }^{29}$ (2002), é baseado na colocação de espirais com potencial trombogênico no interior do ducto arterioso. Vale ressaltar que tal técnica é indicada apenas para casos em que o ducto é de pequeno calibre, sendo impróprio para aqueles maiores que $5 \mathrm{~mm} .^{20}$ Tal intervenção cirúrgica realiza-se por meio da introdução de espirais trombogênicas através de cateter individual direcionados até o ducto por angiografia. Para vários autores, normalmente, é necessária a colocação de 20 espirais para que se tenha uma obliteração completa do ducto arterioso. .,29-32 $^{2}$

As principais desvantagens desta técnica restringem-se ao custo operacional de tal intervenção, já que exige equipamento de angiografia e a aquisição de espirais, que são relativamente onerosos, e ao risco de migração daquelas podendo causar reação vascular iatrogênica a corpo estranho ou embolismo pulmonar ou aórtico potencialmente fatal. ${ }^{5,29}$

\section{Complicações trans e pós-operatórias}

A mais comum complicação cirúrgica é o rompimento do ducto arterioso durante a sua dissecção previamente à ligadura. ${ }^{2,8,24,33}$ Os locais mais propensos ao rompimento do ducto são as junções ducto-aorta, tronco pulmonar-ducto e a porção média do ducto. ${ }^{8}$ Uma vez ocorrida, a hemorragia deve ser controlada rapidamente com pinça vascular, e a junção reparada com sutura. ${ }^{25}$ A técnica de escolha após o rompimento do ducto é a técnica de divisão e sutura do ducto, utilizando fio sintético não-absorvível (polipropileno 6-0). ${ }^{10}$ Usualmente, após rompimento do ducto, simples ligadura não é suficiente, sendo necessário fechamento ou divisão do ducto com sutura manual ou grampo vascular. $^{2}$

Outras complicações cirúrgicas e pós-operatórias são hemorragias não associadas à dissecção do ducto, edema pulmonar, insuficiência cardíaca congestiva, recanalização do ducto, septicemia, fibrilação ventricular e trauma pulmonar iatrogênico. ${ }^{8}$ Vale atentar para o fato de que o fechamento do ducto sem a sua divisão é mais seguro, porém, o risco de recanalização é maior. ${ }^{2}$ 
Pode ocorrer bradicardia durante ligação do ducto arterioso. Caso a freqüência cardíaca caia para menos de 60 bpm, o uso de anticolinérgico deve ser avaliado e empregado. Em casos de hemorragias severas, a transfusão sanguínea deve ser realizada. ${ }^{2,33}$

Independente da técnica adotada recomendase a realização de exame ecocardiográfico de controle para avaliação pós-operatória do êxito da intervenção cirúrgica, ou seja, a não-recanalização do ducto arterioso e atentar para a necessidade ou não de nova intervenção cirúrgica.

\section{Prognóstico}

Os resultados até hoje obtidos indicam que melhor prognóstico para o quadro é alcançado quando a correção cirúrgica é realizada com o animal ainda jovem. ${ }^{10}$

Os principais sintomas apresentados pelo animal cessam após a ligadura do ducto. No pós-operatório, para casos de PDAc, raramente ocorrem intercorrências, sendo de boa evolução e alta sobrevida. No entanto, os casos não corrigidos cirurgicamente tendem a evoluir para quadros de insuficiência cardíaca congestiva esquerda, edema pulmonar e hipertensão pulmonar suprasistêmica, neste caso particular, culminando na reversão do fluxo sanguíneo e provocando severa hipoxemia, cianose e intolerância ao exercício. Uma vez instaurado o quadro de PDAr, a intervenção cirúrgica é contra-indicada. ${ }^{2,10}$

Tem-se que, aproximadamente, 1,5\% dos animais submetidos à ligadura de ducto arterioso persistente sofrem recanalização, sendo necessário, portanto, nova intervenção cirúrgica. É relatada, muito raramente, a sobrevivência de cães com PDAc até a maturidade, embora $70 \%$ dos cães não-tratados cirurgicamente cheguem à óbito antes de um ano de idade. . $^{4,10,29}$

Para cães com PDAr, é citada sobrevida de dois a cinco anos se forem tomados os cuidados clínicos pertinentes ao hematócrito, mantendo níveis sempre entre 62 a $68 \% .^{9}$ O que pode ocorrer, contudo, é o agravamento do quadro de hipoxemia, levando ao óbito repentinamente, devido à formação de trombo arterial ou severas arritmias cardíacas. ${ }^{22}$ Caso lesões na vascularização pulmonar tenham sido estabelecidas, freqüentemente são avançadas e irreversíveis. ${ }^{\prime}$

\section{Considerações Finais}

Valendo-se da experiência junto ao Laboratório de Cirurgia Cardiotorácica do Departamento de Cirurgia da Faculdade de Medicina Veterinária e Zootecnia da Universidade de São Paulo (LCCT/ VCI - FMVZ/USP), São Paulo, SP, diante de tal cardiopatia congênita, cabem algumas considerações. A técnica adotada pelo LCCT é a mesma descrita anteriormente, à exceção da ligadura feita no momento de obstrução do ducto, quando é realizada dupla ligadura com fio de algodão 2-0. Na rotina cirúrgica, observa-se alto índice de sucesso no período pós-operatório de tal intervenção cirúrgica, alcançando $92 \%$ de sobrevida, valor semelhante ao encontrado na literatura. Cumpre ressaltar, entretanto, intercorrências em dois casos clínicos da cardiopatia ora referida que evoluíram para o óbito em ambos os cães. O primeiro decorrente à falha de diagnóstico, quando não se identificou a priori como sendo um caso de PDAr, e após a ligadura do ducto arterioso o animal veio à óbito. No segundo animal houve rompimento do ducto arterioso persistente na região média durante dissecção, e sendo o paciente de pouca massa corpórea, $5 \mathrm{~kg}$, não houve tempo suficiente para deter o quadro hemorrágico advindo choque hipovolêmico irreversível.

Vale ressaltar que, apesar de a correção cirúrgica ser altamente exeqüível, deve ser realizada por equipe com experiência em cirurgia torácica, a fim de se evitar o surgimento de conseqüências danosas e, mesmo que tais ocorram, possam se proceder às condutas mais adequadas para corrigi-las. 


\section{Abstract}

Objective: To perform a literature review on patent ductus arteriosus, pointing out the main morphological, physiopathological, clinical, diagnostic and prognostic aspects, as well as possible treatments. Data Sources: CAB Abstracts 1990-2002; MEDLINE 1978-2002. Data Synthesis: Patent ductus arteriosus (PDA) is the most common congenital cardiac disease in dogs. The most common type is the left-to-right shunt, although the reverse PDA may sometimes occur, that is, with inversion of the blood flow through the duct. Conclusions: The joint understanding of all these aspects improves the comprehension of the phenomena involved in the clinical course of the disease, giving a crucial contribution to both, treatment indication and animal recovery.

Keywords: Heart defect congenital. Ductus arteriosus patent. Thoracic surgery. Dogs.

\section{Resumen}

Objetivo: Presentar una revisión de la literatura acerca de la persistencia del conducto arterioso, levantando los principales aspectos morfológicos, fisiopatológicos, clínicos, del diagnóstico y pronóstico y los posibles tratamientos. Fuentes Consultadas: CAB Abstracts 1990-2002; MEDLINE 1978-2002. Síntesis de los Datos: La persistencia del conducto arterioso (PCA) es la lesión cardiaca congénita encontrada con mayor frecuencia en perros. La forma más común se caracteriza por el desvío sanguíneo de izquierda a derecha. Más raramente aparece la PCA revertida, con inversión del flujo a través del conducto. Conclusiones: La consideración global de todos estos aspectos ayuda a la comprensión de los fenómenos envueltos en la evolución clínica de la afección, contribuyendo de manera decisiva para la indicación del tratamiento adecuado y la recuperación del animal.

Palabras-clave: Cardiopatías congénitas. Persistencia del conducto arterioso. Cirugía torácica. Perros.

\section{Referências}

1. COTE, E.; ETTINGER, S. J. Long-term clinical management of right-to-left (reversed) patent ductus arteriosus in 3 dogs. Journal of Veterinary Internal Medicine, v. 15, p. 39-42, 2001.

2. FOSSUM, T. W. Surgery of the cardiovascular system. In: . Small animal surgery. Saint Louis: Mosby-Year Book, 1997. p. 575-608.

3. JONES, C. L.; BUCHANAN, J. W. Patent ductus arteriosus: anatomy and surgery in a cat. Journal of the American Veterinary Medical Association, v. 179 , p. $364-369,1981$.
4. OSWALD, G. P.; ORTON, E. C. Patent ductus arteriosus and pulmonary hypertension in related Pembroke Welsh corgis. Journal of the American Veterinary Medical Association, v. 202, p. 761-764, 1993.

5. SNAPS, F. R. et al. Treatment of patent ductus arteriosus by placement of intravascular coils in a pup. Journal of the American Veterinary Medical Association, v. 207, p. 724-725, 1995.

6. TIDHOLM, A. Retrospective study of congenital heart defects in 151 dogs. Journal of Small Animal Practice, v. 38, p. 94-98, 1997. 
7. ACKERMAN, N. et al. Patent ductus arteriosus in the dog: a retrospective study of radiographic, epidemiologic, and clinical findings. American Journal of Veterinary Research, v. 39, p. 1805 $1810,1978$.

8. BIRCHARD, S. J.; BONAGURA, J. D.; FINGLAND, R. B. Results of ligation of patent ductus arteriosus in dogs: 201 cases (1969-1988). Journal of the American Veterinary Medical Association, v. 196, p. 2011-2013, 1990.

9. BONAGURA, J. D. Moléstia cardíaca congênita. In: ETTINGER, S.J. Tratado de medicina interna veterinária: moléstias do cão e do gato. São Paulo: Manole, 1992. p. 1026-1082.

10. EYSTER, G. E. Basic cardiac surgical procedures. In: SLATTER, D. Textbook of small animal surgery. 2. ed. Philadelphia: Saunders, 1993. v. 1, p. 893-918.

11. HOLT, D. et al. Esophageal obstruction caused by a left aortic arch and an anomalous right patent ductus arteriosus in two German Shepherd littermates. Veterinary Surgery, v. 29, p. 264-270, 2000.

12. McENTEE, K. et al. Clinical vignette: tetralogy of Fallot associated with a patent ductus arteriosus in a German shepherd dog. Journal of Veterinary Internal Medicine, v. 12, p. 53-55, 1998.

13. FERNANDEZ DEL PALACIO, M. J. et al. Persistent left cranial vena cava associated with multiple congenital anomalies in a six-week-old puppy. Journal of Small Animal Practice, v. 38, p. $526-530,1997$.

14. ETTINGER, S. J.; SUTER, P. F. Congenital heart disease. In: Canine cardiology. Philadelphia: Saunders, 1970. p. 497-602.

15. PYLE, R. L. et al. Patent ductus arteriosus with pulmonary hypertension in the dog. Journal of the American Veterinary Medical Association, v. 178 , p. 565-571, 1981.
16. DE REEDER, E. G. et al. Ultrastructural and immunohistochemical changes of the extracellular matrix during intimal cushion formation in the ductus arteriosus of the dog. Atherosclerosis, v. 79, p. 29-40, 1989.

17. GitTenberger DE GROOT, A. C. et al. Histologic studies on normal and persistent ductus arteriosus in the dog. Journal of the American College of Cardiology, v. 6, p. 394-404, 1985.

18. van ISRAEL, N. et al. Hemolysis associated with patent ductus arteriosus coil embolization in a dog. Journal of Veterinary Internal Medicine, v. 15, p. 153-156, 2001.

19. O'BRIEN, S.E. et al. Right-to-left patent ductus arteriosus with dysplastic left ventricle in a dog. Journal of the American Veterinary Medical Association, v. 192, p. 1435-1438, 1988.

20. SAUNDERS, J. H. et al. Use of a balloon occlusion catheter to facilitate transarterial coil embolisation of a patent ductus arteriosus in two dogs. Veterinary Record, v. 145, p. 544-546, 1999.

21. HENDERSON, R. A.; JACKSON, W. F. Coração e grandes vasos. In: BORJRAB, M. J. Cirurgia dos pequenos animais. Botucatu: ROCA, 1983. p. 491-522.

22. ARORA, M. Reversed patent ductus arteriosus in a dog. Canadian Veterinary Journal, v. 42, p. 471472, 2001.

23. MOISE, N. S. Doppler echocardiographic evaluation of congenital cardiac disease: an introduction. Journal of Veterinary Internal Medicine, v. 3, p. 195-207, 1989.

24. DOWNS, M. O.; STAMPLEY, A. R.; RAWLINGS, C. A. A wire loop technique for ligation of patent ductus arteriosus. Journal of Small Animal Practice, v. 36, p. 489-491, 1995. 
25. HUBER, E.; MONTAVON, P. M. Patent ductus arteriosus in a dog: modified method of double ligation. Schweizer Archiv fur Tierheilkunde, v. 134, p. 41-6, 1992.

26. FANTONI, D. T. Anestesia no cardiopata. In: FANTONI, D. T.; CORTOPASSI, S. R. G. Anestesia em cães e gatos. São Paulo: Roca, 2002. p. 294-320.

27. GOODWIN, J. K. Cardiopatias congênitas. In: TILlEY, L. P.; GOODWIN, J. K. Manual de cardiologia para cães e gatos. São Paulo: Roca, 2002. p. $259-276$.

28. MOORE, K. W.; STEPIEN, R. L. Hydroxyurea for treatment of polycythemia secondary to right-to-left shunting patent ductus arteriosus in 4 dogs. Journal of Veterinary Internal Medicine, v. 15, p. $418-421,2001$.

29. SCHNEIDER, M. et al. Transvenous embolization of small patent ductus arteriosus with single detachable coils in dogs. Journal of Veterinary Internal Medicine, v. 15, p. 222-228, 2002.

30. FELLOWS, C. G. et al. Treatment of patent ductus arteriosus by placement of two intravascular embolisation coils in a puppy. Journal of Small Animal Practice, v. 39, p. 196-199, 1998.

31. GLAUS, T.M. et al. Closure of a persistent ductus arteriosus of Botallo in two dogs using transarterial coil embolization. Schweizer Archiv fur Tierheilkunde, v. 141, p. 191-194, 1999.

32. TANAKA, R. et al. Detachable coils for occlusion of patent ductus arteriosus in 2 dogs. Veterinary Surgery, v. 30, p. 580-584, 2001.

33. HUNT, G. B. et al. Intraoperative hemorrhage during patent ductus arteriosus ligation in dogs. Veterinary Surgery, v. 30, p. 58-63, 2001.

Endereço / Address / Dirección:

Prof. Dr. Angelo João Stopiglia

Laboratório de Cirurgia Cardiotorácica

Departamento de Cirurgia

Faculdade de Medicina Veterinária e Zootecnia

Universidade de São Paulo

Av. Prof. Dr. Orlando Marques de Paiva, 87

CEP: 05508-000 - São Paulo, SP, Brasil

Tel: 0XX (11) 3091-9953

Recebido em: 06/08/2004

E-mail: stopa@usp.br 